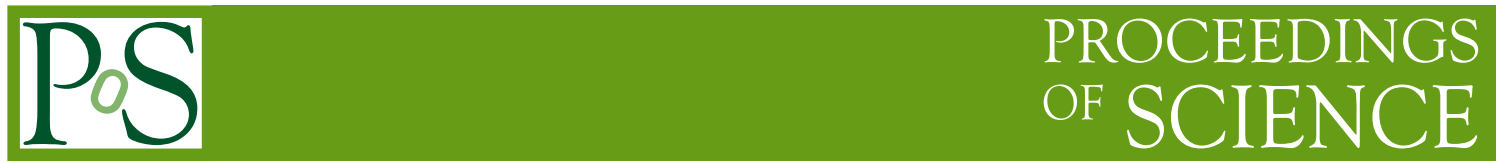

\title{
On the Quantum Disc and the Equivalence of Morse-Smale Systems
}

\author{
Antonio R. da Silva* \\ Department of Mathematics \\ Federal University of Rio de Janeiro,Brazil \\ E-mail:ardasilva@openlink.com.br
}

\section{Franciscus J. Vanhecke}

Department of Physics

Federal University of Rio de Janeiro,Brazil

E-mail: vanheckedif.ufrj.br

\section{Cassio Sigaud}

Department of Physics

Federal University of Rio de Janeiro, Brazil

E-mail: sigaudeif.ufrj.br

In this short paper we show that Connes'quantization of a Morse-Smale vector field on an orientable two-dimensional closed manifold satisfies Berezin's requirement of equivalence preservation.

Fourth International Winter Conference on Mathematical Methods in Physics 09 - 13 August 2004

Centro Brasileiro de Pesquisas Fisicas (CBPF/MCT), Rio de Janeiro, Brazil

\footnotetext{
*Speaker.
} 


\section{Introduction and Berezin's concept of quantization}

According to Berezin [1] a mathematical quantization of a sympletic manifold should be a family of associative algebras with involution satisfying certain properties. Among these properties is a functorial one, that says that equivalence should be preserved under quantization. A typical example is Berezin's quantum disc, where given $U$ unit disc in complex plane, with its canonical sympletic form $w=\frac{i}{2}\left(1-|z|^{2}\right)^{-2} d z d \bar{z}$ and the corresponding Poisson bracket $\{f, g\}=$ $i\left(1-|z|^{2}\right)^{2}(\partial f \bar{\partial} g-\bar{\partial} f \partial g)$. One builds a quantization of $U$ by considering the family of all square integrable functions with respect to the measures $m_{h}=\frac{i}{2}\left(1-|z|^{2}\right)^{\frac{1}{h}-1} d z d \bar{z}$ endowed with the inner product $\langle f, g\rangle_{h}=\frac{i}{2} \int_{U} f(z) \overline{g(z)}\left(1-|z|^{2}\right)^{\frac{1}{h}-1} d z d \bar{z}, \quad 0<h<1$, which generates a family of Hilbert spaces with corresponding norms $\|f\|_{h}=\left\{\frac{i}{2} \int_{U}|f(z)|^{2}\left(1-|z|^{2}\right)^{\frac{1}{h}(-1)} d z d \bar{z}\right\}^{1 / 2}$. These spaces have orthonormal basis given by $\varphi_{n, h}(z)=\left\{\frac{1}{\pi} \frac{\Gamma\left(h^{-1}+n+1\right)}{n ! \Gamma\left(h^{-1}\right)}\right\}^{1 / 2} z^{n}$. Setting $T_{\varphi}\left(f(z)=\left(P M_{\varphi} f\right)(z)\right.$, where $M_{\varphi}(f)=\varphi f$ and $P$ is the orthogonal projection on the subspace of holomorphic functions one gets the corresponding Bergman kernels $K_{h}(z, w)=\frac{1}{h \pi} \frac{1}{(1-z \bar{w})^{1+1 / h}}$ then the algebras generated by the operators $T_{\varphi}$ form the quantum disc. Actually, these algebras coincide with the algebras generated by the deformations $[z, \bar{z}]=h(I-z \bar{z})(I-\bar{z} z)$ (see [6]). In general, the Berezin quantization of a sympletic manifold $(M, w)$ is given by a family of associative algebras $\mathcal{A}=\left(A_{h}\right)_{h \in E}$, where $E$ denotes the set of positive real numbers having 0 as a limit point, such that the correspondence principle hold, that is, there is a family of involution preserving linear mappings $T^{(h)}: C^{\infty}(M) \rightarrow A_{h}$ satisfying $\lim _{h \rightarrow 0}\left\|\frac{1}{i h}\left[T_{\varphi}^{(h)}, T_{\psi}^{(h)}\right]-T_{\{\varphi, \psi\}}\right\|=0$, where $\{\cdot, \cdot\}$ is the Poisson bracket associated to $w$. Further, the correspondence $(M, w) \rightsquigarrow \mathcal{A}$ must satisfy

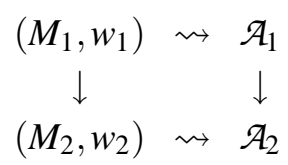

These properties hold in the special case of the quantum disc(see [6]). Berezin's conceptual treatment of quantization has been extended in many directions. Recently, Kontsevich proved that every finite-dimensional Poisson manifold, that is, any manifold $M$ with a Poisson bracket on $C^{\infty}(M)$, always admits a deformation quantization. In the early eighties, by using his then new noncommutative integration theory, A. Connes quantized the dynamics described by regular foliations by establishing a correspondence $\mathcal{F} \rightsquigarrow C^{*}(\mathcal{F})$, between regular foliations and $C^{*}$-algebras. Soon after, Sheu extended this construction to include singular foliations. Our purpose here is to show that Sheu's construction, may be used to produce a quantization of a Morse-Smale vector field on a closed, that is, compact without boundary, orientable two-dimensional manifold satisfying Berezin's requirements. Here, by regular foliations is meant the ones that have leaves with the same dimension, while singular foliations are the ones with leaves having different dimensions. 


\section{Connes' foliation algebra and Sheu's extension}

We first recall Connes' basic construction in a special case. Let be given the first-order system

$$
\left\{\begin{array}{l}
\frac{d x}{d t}=u \\
\frac{d u}{d t}=f(t, x, u)
\end{array}\right.
$$

By plotting its trajectories one gets a natural 1-dim foliation of $\mathbb{R}^{3}$. Associated to this foliation one has its graph $\Omega(\mathcal{F})$ defined as the subset of $\mathbb{R}^{6}$ consisting of points $\left(t_{1}, x_{1}, u_{1}, t_{2}, x_{2}, u_{2}\right)$ such that there is a trajectory through $\left(t_{1}, x_{1}, u_{1}\right)$ and $\left(t_{2}, x_{2}, u_{2}\right)$. This manifold carries a natural, partially defined, group like structure (groupoid). It was precisely through this notion of groupoid that Connes introduced a quantization process. By a groupoid $G$ with basis $B$ we understand a set $G$ endowed with mappings $r: G \rightarrow B, \quad s: G \rightarrow B$, called target and source maps and a partially defined binary operation $(x, y) \mapsto x y$ such that: (i) $x y$ is defined whenever $r(y)=s(x)$; (ii) associativity holds; (iii) for each $x \in G$ there is a left neutral element $r_{x}$ and a right neutral element $s_{x}$ such that $r_{x} \cdot x=x=x \cdot s_{x}$. Thus instead of the unity of the group we have the unit space $G^{o}:=\left\{x x^{-1}: x \in G\right\}$. Groups are groupoids with $G^{o}=\{e\}$. The mappings $r, s: G \rightarrow G^{o}$, with $r(x)=x x^{-1}$ and $s(x)=x^{-1} x$ are naturally associated to $G$ and allow us to identify $B$ with $G^{o}$. By a topological groupoid we mean a groupoid $G$ endowed with a topology such that the multiplication and the inverse mappings, whenever defined, are continuous. Let us assume, at first, that $G$ is locally compact, Hausdorff and that the topology on $G$ is second countable. It is well-known that in case $G$ is a group with a left Haar measure $d x, L^{1}(G, d x)$ can be made into a *-Banach algebra with multiplication $(f * g)(x)=\int f(y) g\left(y^{-1} x\right) d y$ and the involution: $f^{*}(x)=\overline{f\left(x^{-1}\right)} \Delta(x)^{-1}$, where $\Delta$ is the modular function of $G$. The group $C^{*}$-algebra $C^{*}(G)$ is defined to be the completion of $L^{1}(G)$ with respect to the norm $\|f\|_{C^{*}}=\sup \{\|\pi(f)\|: \pi$ is a unitary representation of $G\}$, where $\pi(f)=\int_{G} f(x) \pi(x) d x$ and \|\| is the operator norm for operators on the Hilbert space of $\pi$.

In a locally compact groupoid one does not have a Haar measure, so the above procedure can not be used to construct a $C^{*}$-algebra out of it. Connes introduced the following substitute for a Haar measure: A transverse function on a locally compact groupoid is a family of measures $\left\{\lambda^{x}: x \in G^{o}\right\}$ such that: (i) the support of $\lambda^{x}$ is contained in $G^{x}:=r^{-1}(x)$; (ii) for each $f \in C_{c}(G)$ the mapping $\lambda(f): x \rightarrow \int f d \lambda^{x}$ is continuous; (iii) for each $\gamma \in G_{x}^{y}:=r^{-1}(y) \cap s^{-1}(x)$ and each $f \in C_{c}(G)$ holds $\int f\left(\gamma^{\prime}\right) d \lambda^{x}\left(\gamma^{\prime}\right)=\int f\left(\gamma^{\prime}\right) d \lambda^{y}\left(\gamma^{\prime}\right)$. The support of the mapping $\lambda: C_{c}(G) \rightarrow C_{c}\left(G^{o}\right)$ defined by $\lambda(f)(x)=\int f d \lambda^{x}$ is the support of the transverse function. The transverse function is called a Haar system when its support is $G^{o}$. It does not have to exist in a general groupoid, and even if it does, it is usually not unique. Given a foliated manifold $(M, \mathcal{F})$ the holonomy groupoid or graph of $(M, \mathcal{F})$ is the groupoid with $M$ as its sets of objects and with the set $G$ of morphisms defined by the properties: a. there is no morphism $x \rightarrow y$ unless $x$ and $y$ lie on the same leaf $L$ of $\mathcal{F}$; b. the portion $r^{-1}(L)=s^{-1}(L)$ of $G$ over a leaf $L$ is a quotient of the fundamental groupoid of $L$, and c. two homotopy classes $\left[\gamma_{1}\right],\left[\gamma_{2}\right]$ of paths from $x$ to $y$ in a leaf $L$ are identified in $G$ iff they have the same holonomy, i.e., if following the normal structure of $\mathcal{F}$ along $\gamma_{1}$ and $\gamma_{2}$ yields the same germ of a homeomorphism from a transverse $q$-disk at $x$ to a transverse $q$-disk at $y$. One can 
give $G$ a natural structure of a $(2 p+q)$-manifold. If the leaves of $\mathcal{F}$ are all simply connected, of more generally, without holonomy, then $G$ reduces to the groupoid of an equivalence relation $R$ on $M, \quad R$ being the relation of "lying on the same leaf" as in case of the 1-dim foliation of $\mathbb{R}^{3}$ given previously. In general, given a locally compact groupoid with a Haar system $(G, \lambda)$. We can define a ${ }^{*}$-algebra structure of $C_{c}(G)$, taking $(f * g)(\gamma)=\int f\left(\gamma^{\prime}\right) g\left(\gamma^{-1} \gamma\right) d \lambda^{s(\gamma)}\left(\gamma^{\prime}\right)$; and $f^{*}(\gamma)=\overline{f\left(\gamma^{-1}\right)}$. The $C^{*}$-algebra envelope of this *-algebra is the $C^{*}$-algebra of the groupoid $G$. We define groupoid equivalence the following way: Let $G$ be a groupoid. Consider a fiber bundle with base space $G^{o}$, total space $Z$ and projection $\pi$. Given $x, y \in G^{o}$, each $\gamma \in G_{x}^{y}=r^{-1}(y) \cap s^{-1}(x)$ induce a bijection: $L_{\gamma}: \pi^{-1}(x) \rightarrow \pi^{-1}(y)$ which satisfies: $L_{\gamma^{\prime}}=L_{\gamma} \circ L_{\gamma^{\prime}}$. A pair $(\gamma, z)$ is said compatible if $\pi(z)=s(\gamma)$ and the product of $\gamma$ by $z$ is defined by $\gamma \cdot z=L_{\gamma}(z)$. The set of compatible pairs is denoted by $G * Z$. If $\pi$ is open and continuous, and the product map $G * Z \subset G \times Z \rightarrow Z$ is continuous then $Z$ is called a left $G$-space. Similarly one defines right $G$-spaces. Given two groupoids $G$ and $G^{\prime}$ we define a $\left(G, G^{\prime}\right)$-space as a topological space $Z$ that satisfies: (i) $Z$ is a left $G$-space; (ii) $Z$ is right $G^{\prime}$-space; (iii) the actions of $G$ and $G^{\prime}$ on $Z$ commute. We say that two groupoids $G$ and $G^{\prime}$ are equivalent whenever there is a $\left(G, G^{\prime}\right)$-space such that: (i) if $\pi\left(z^{\prime}\right)=\pi(z)$ then there is a unique $\gamma \in G$ such that $z^{\prime}=\gamma z$. (ii) if $\pi^{\prime}\left(z^{\prime}\right)=\pi^{\prime}(z)$ then there is a unique $\gamma^{\prime} \in G^{\prime}$ and that $z^{\prime}=z \gamma^{\prime}$.

\section{3. $C^{*}$-algebras equivalence and the main result}

Let $A$ be a $C^{*}$-algebra and $X$ be a (right) $A$-module. We define a (right) inner product in $X$ as the mapping $\langle\cdot, \cdot\rangle_{A}: X \times X \rightarrow A$ such that (i) $\langle x, \lambda y+\mu z\rangle_{A}=\lambda\langle x, y\rangle_{A}+\mu\langle x, z\rangle_{A}$; (ii) $\langle x, y \cdot a\rangle_{A}=\langle x, y\rangle_{A} \cdot a$; (iii) $\langle x, y\rangle_{A}^{*}=\langle y, x\rangle_{A}$; (iv) $\langle x, x\rangle_{A} \in A^{+}$; (v) $\langle x, x\rangle=0 \Rightarrow x=0$. Further, $\|x\|_{A}:=\left\|\langle x, x\rangle_{A}\right\|^{1 / 2}$.

By an A-Hilbert module $X$ we mean an $A$-module with inner product that is complete with respect to $\|\cdot\|_{A}$. We say $X$ is saturated whenever $I:=\operatorname{span}\left\{\left(\langle x, y\rangle_{A} ; x, y \in X\right\}\right.$ is dense in $A$. Now, let $A$ and $B$ be $C^{*}$-algebras. As $A-B$ imprimitive bimodule $X$ is an $A-B$ module such that: (i) $X$ is saturated left $A$ (resp. right $B$ )-Hilbert module; (ii) $\langle a \cdot x, y\rangle_{B}=\left\langle x, a^{*} y\right\rangle_{B}$ and ${ }_{A}\langle x \cdot b, y\rangle=_{A}\left\langle x, y \cdot b^{*}\right\rangle$; (iii) ${ }_{A}\langle x, y \cdot z\rangle=x \cdot\langle y, z\rangle_{B}$.

Two $C^{*}$-algebras $A$ and $B$ are then said Morita equivalent if there exists an $A-B$ imprimitivity bimodule that implements the equivalence between $A$ and $B$.

Now let $(V, \mathcal{F})$ be a singular foliation and the subsets $F$ in $F$ leaves. An $s$-density on an $n$-dimensional vector space $V$ over $\mathbb{R}$ is a map $\alpha: \Lambda^{n} V^{*} \sim\{0\} \rightarrow \mathbb{C}$ such that for any non-zero $c \in \mathbb{R}$ and non-zero $z \in \Lambda^{n} V^{*}$, we have $\alpha(c z)=|c|^{s} \alpha(z)$. Let $\Omega^{s} V$ be the set of $s$-densities on $V$; $\Omega^{s}(V) \Omega^{t}(V) \subset \Omega^{s+t}(V) ; \quad \Omega^{*} V$ is a vector space over $\mathbb{C}$ under pointwise operations; $\Omega^{s} V$ is onedimensional: Fix a non-zero $\omega \in \Lambda^{n} V$ and $\lambda \in \mathbb{C}$. Let $\left\{e_{i}\right\}$ be a basis for $V$ with dual basis $\left\{e_{i}^{*}\right\}$. Then for $c \in \mathbb{R} \sim\{0\}$, define $\alpha$ by: $\alpha\left(c e_{1}^{*} \wedge \cdots \wedge e_{n}^{*}\right)=\lambda|\omega|\left|\left(c e_{1}^{*}, \ldots, e_{n}^{*}\right)\right|^{s}$ we write $\alpha=\lambda|\omega|^{s}$. A $s$-density $\alpha$ is said to be positive if $\alpha(\omega) \geq 0, \forall \omega$.

Let $(V, \mathcal{F})$ be a singular foliation and $\mathcal{G}$ be its holonomy groupoid. Let $V_{m}$ be the union of leaves of dimension $m$. Then on $V_{m}$ we have a smooth positive half line bundle, $\left|\Lambda^{m} \mathcal{F}\right|$, the bundle of one-densities along the leaves in $V_{m}$. By fixing a smooth section $\mu_{m}$ of $\left|\Lambda^{m} \mathcal{F}\right|$, the union of the half line bundles $\left|\Lambda^{m} \mathcal{F}\right|$ becomes a trivial half line bundle $|\Lambda \mathcal{F}|$ over $V$. Let $s$ and $t$ be the source and target maps defined on $\mathcal{G}$. For any $p \in \mathcal{G}$ we can find neighborhoods $U$ and $W$ of $s(p)$ and $t(p)$ respectively such that $p$ is an one-to-one correspondence between the leaf spaces $U / U \cap \mathcal{F}$ 
and $W / W \cap \mathcal{F}$. We define $G(U, p, W)$ to be the subset $\{(x, y) \mid x \in U, y \in W$ and $p([x])=[y]\}$ of $U \times W$. Then such $G(U, p, W)$ 's form a fundamental system of open sets of $\mathcal{G}$. Now we construct the foliation algebra of $\mathcal{F}$ in the following way. Let $G(U, p, W)_{m}=G(U, p, W) \cap s^{-1}\left(V_{m}\right)$ (union of leaves of dimension $m$ ).

Define $\Lambda(U, p, W)$ to be the set of bounded complex valued functions $\xi$ on $G(U, p, W)$ satisfying the following conditions: (1) $\xi$ is continuous on each $G(U, p, W)_{m}$. (2) the closure of $\operatorname{supp}(\xi)$ in $G(U, p, W)$ is compact; (3) under convolution with respect to the fixed measure $\mu, \xi$, $\xi^{\prime}$ preserve continuous functions on $V$, i.e., $\xi * f \in C(V), \forall f \in C(V)$ and $\xi^{*} * g \in C(V), \forall g \in C(V)$ where $\xi^{*}(x, y):=\overline{\xi(y, x)}$; (4) $\|\xi\|_{1}<\infty$, where $\|\xi\|_{1}=\max \left\{\int^{x}|\xi(x, y)| \mu(x), \int^{y}|\xi(x, y)| \mu(y)\right\}_{x \in U, y \in W}$. Let $\mathcal{A}(\mathcal{G}, \mu)$ be the linear span of elements in such $\Lambda(U, p, W)$ 's. $\mathcal{A}(\mathcal{G}, \mu)$ is an involutive algebra normed by $\|\xi\|_{1}:=\max \left(\int_{t(p)=y}|\xi(p)|\left(x^{s}(\mu)\right)(p), \int_{s(p)=x}|\xi(p)|\left(t^{*}(\mu)\right)(p)\right)$. For each leaf $F$, the left convolution (with respect to $\mu$ ) gives rise to a regular representation $\pi_{F}$ of $\mathcal{A}(\mathcal{G}, \mu)$ on the Hilbert space $L^{2}(F, \mu)$ of $\mu$-square integrable functions on $F, \quad\left\|\pi_{F}(\xi)\right\| \leq\|\xi\|_{1}$. The algebra $\mathcal{A}(\mathcal{G}, \mu)$ normed by $\|\xi\|=\sup _{F \in \mathcal{F}}\left\|\pi_{F}(\xi)\right\|$ is a pre- $C^{*}$-algebra whose completion $C^{*}(F, \mathcal{F}, \mu)$ is called a (reduced) foliation $C^{*}$-algebra of $(V, \mathcal{F})$.

We recall that given a vector field $X$ on a manifold $M$, that is $X: M \rightarrow T M$, where $T M$ is the tangent bundle over $M$. The integral curves of the system $\dot{x}(t)=X(x(t)), x(0)=p$ define a flow $\mathcal{F}_{t}(p)=\mathcal{F}(t, p)$. Taking $p \in M$ the orbit through $p$ is defined by $O(p)=\left\{\mathcal{F}_{t}(p) \in M: t \in I\right\}$. Now given two vector fields $X$ and $Y$ on $M$ with corresponding flows $\mathcal{F}$ and $\mathcal{G}$, respectively, we say $X$ and $Y$ are topologically equivalent whenever there is an homeomorphism $h: M \rightarrow M$ that maps orbits of $X$ into orbits of $Y$ preserving their orientation. Further, we say that $X \in \mathfrak{X}^{r}(M)$ is structurally stable whenever there is a neighborhood $\mathcal{V}$ of $X$ in $\mathfrak{X}(M)$ such that every vector field $Y \in \mathcal{V}$ is topologically equivalent to $X$. In other words, the topological behaviour of the orbits of $X$ does not change under small perturbations. Suppose now that $M$ is a 2-dimensional closed manifold. Then $X \in \mathfrak{X}^{r}(M)$ is called Morse-Smale if: (a) the vector field $X$ has a finite number of hyperbolic singular points and a finite number of hyperbolic periodic trajectories; (b) there is no saddle connection, that is, no separatrix joining one saddle to another or to itself; (c) any orbit has a unique $\alpha$-limit as well as a unique $\omega$-limit.

Morse-Smale vector fields on compact two-dimensional manifolds were proved to be abundant and were classified through important contributions due to Peixoto (for a modern approach, using the technique of atoms and molecules, see [2]). Among his results he proved that: For a closed orientable 2-manifold one has: (i) a vector field $X \in \mathfrak{X}^{r}$ is structurally stable iff it is Morse-Smale; (ii) the set $\Sigma$ of all Morse-Smale vector fields is open and dense in the space $\mathfrak{X}^{r}$; (iii) two distinguished graphs correspond to equivalent flows iff they are isomorphic.

Our main result reads:

Theorem If $X$ and $Y$ are topologically equivalent Morse-Smale vector fields on a closed orientable 2-manifold $M$. Then the associated $C^{*}$-algebras $C^{*}\left(\mathcal{F}_{i}\right)$ are equivalent as $C^{*}$-algebras, that is, they are Morita equivalent.

The steps of the proof consists of showing that the foliations associated to the equivalent Morse-Smale vector fields $X$ and $Y$ are equivalent. Further, since such foliations are (étale) (see [5]) their holonomy groupoids are equivalent as groupoids. On the other hand, a result of Muhly, 
Renault and Williams (see [7]) show that the $C^{*}$-algebras associated to equivalent groupoids must be Morita equivalent. The above result shows that Connes'quantization of a Morse-Smale vector field satisfies Berezin's requirement of equivalence preservation.

\section{References}

[1] F.A. Berezin, General concept of quantization, Comm. Math. Phys. 40 (1975) 153.

[2] A.V. Bolsinov and A.F. Fomenko, Integrable Hamiltonian Systems, Chapman and Hall/CRC, Boca Raton 2004.

[3] A. Connes, Noncommutative Geometry, Academic Press, New York 1994.

[4] C. Debord, Holonomy Groupoids of Singular Foliations, J. Differential Geometry 58 (2001) 467.

[5] A. Haefliger, Groupoids and Foliations,Contemporary Mathematics282 (2001) 83.

[6] S. Klimek and A. Lesniewski, Quantum Riemann Surfaces.Part I, Comm. Math. Phys.146 (1992) 103.

[7] P. Muhly, J. Renault and F. Williams, Equivalence and isomorphism for groupoid $C^{*}$-algebras, $J$. Operator Theory, 17 (1987) 809.

[8] M.M. Peixoto, On the classification of flows on 2-manifolds, in Proc. Symp. Dynamical Systems (University of Bahia, Salvador, Brasil), edited by M.M. Peixoto, Academic Press, New York (1973) 389.

[9] A. Scheu, Singular Foliation C*-algebras, Proc. Amer. Math. Soc. 1044 (1988) 1197.

[10] F.J. Vanhecke, C. Sigaud and A.R. da Silva, Noncommutative Configuration Space. Classical and Quantum Mechanical Aspects, to appear. 